\title{
Wolfram Ette
}

\section{Prosa als Wirklichkeitsverhältnis}

\author{
Ein Kommentar zu Erich Auerbachs Mimesis
}

\section{Eine Literaturgeschichte der Prosa}

Eines der Schlüsselkapitel von Erich Auerbachs Mimesis ist die Abhandlung über Dante. Dante stellt, so Auerbach, das letzte Aufbäumen des christlichen Mittelalters dar; hier gelangt die für den christlichen Realismus von Antike und Mittelalter so bezeichnende Denkfigur der Figuraldeutung, nach der in der Idee des absoluten Gottes noch die nichtigste Singularität irdischer Existenz aufgehoben ist, ja die Unterscheidung von ,wichtig‘ und ,unwichtig، (und damit die antike Stiltrennungsregel) keinerlei Bedeutung mehr besitzt, zum Höhepunkt und Abschluss. In triumphaler Selbstübersteigerung der Beziehung von zeitlichem Dasein und ewig-heilsgeschichtlicher ,Figur projiziert Dante schlechterdings alles, jede nur denkbare irdische Erfahrung in sein Jenseits; Hölle, Fegefeuer und Paradies absorbieren virtuell die gesamte Erfahrungswelt des von Begehren und Schuldgefühlen vorangetriebenen Erzählers, der wiederum, hochempfindlich und berührbar, all das Gesehene in sich aufnimmt und erzählerisch verarbeitet. In dieser überspannten Gleichung, in der alles ins Jenseits und von dort aus alles ins Ich fällt, wird, so Auerbach, der Rahmen der Figuraldeutung letztendlich „Zerstört“: „Dantes Werk verwirklichte das christlich-figurale Wesen des Menschen und zerstörte es in der Verwirklichung selbst; der gewaltige Rahmen zerbrach durch die Übermacht der Bilder, die er umspannte. "1 Auf diese Weise wird Dante zwar nicht zum Ausgangspunkt des säkularen literarischen Realismus, dem die folgenden Kapitel von Mimesis gewidmet sind, aber zu dem Umschlagspunkt, an dem das theologische Denken des Mittelalters mit seinem ihm ganz eigentümlichen Realismus, einen point of no return erreicht. Es wurde übererfüllt und in der Erfüllung zerbrochen, damit säkulare Wirklichkeitsdarstellungen auf den Weg gebracht werden konnten. „Dantes große Kunst treibt es so weit, daß die Wirkung ins Irdische umschlägt, und in der Erfüllung die Figur den Hörenden allzusehr ergreift; das Jenseits wird zum Theater des Menschen und seiner Leidenschaften.“2

Freilich hat Dantes Realismus eine ihm ganz eigentümliche Grenze. Die Welt der Erfahrung kann nämlich von Dante allein als Serie hochexpressiver Gesten dargestellt werden. Die Commedia besteht, erzählerisch gesehen, aus einer Aufeinanderfolge

1 Erich Auerbach: Mimesis. Dargestellte Wirklichkeit in der abendländischen Literatur, Tübingen/ Basel 2001, 193.

2 Ebd., 192.

Ә Open Access. ( 2021 Wolfram Ette, publiziert von De Gruyter.

(c) BY-ND Dieses Werk ist lizenziert unter der Creative Commons Attribution-NoDerivatives 4.0 
mehr oder weniger traumatischer Schocks. ${ }^{3}$ Die Erzählung des Jenseitsgedichts reiht isolierte Bilder aneinander; und die unerhörte Kunst Dantes besteht eben darin, die harten Schnitte von Bild zu Bild einerseits zuzulassen, andererseits in großartig abgestuften syntaktischen Konstruktionen, die es in dieser Form in der Volkssprache noch nicht gegeben hatte, aufzufangen und in eine einzige, große Bewegung zu verwandeln.

Dante verfügt über Stilmittel von einem Reichtum, wie sie keine europäische Vulgärsprache vor ihm kannte [es folgt eine stilistische Analyse der von Auerbach zitierten Episode]. Gegenüber all den Früheren [...] besitzt sein Ausdruck so unvergleichlich mehr Reichtum, Kraft und Biegsamkeit, er kennt und verwendet so unvergleichlich mehr Formen, er faßt die verschiedensten Erscheinungen mit so unvergleichlich sicherem und festem Griff, daß man zu der Überzeugung gelangt, dieser Mensch habe die Welt durch seine Sprache neu entdeckt. ${ }^{4}$

Die exemplarische Episode, an der Auerbach das demonstriert, findet sich im zehnten Gesang des Infernos. Es sind hier namentlich zwei Schnitte, auf die er hinweist - einmal das urplötzliche Erscheinen des Farinata, der das Gespräch zwischen Vergil und dem Erzähler unterbricht, um diesen nach den politischen Entwicklungen in der Toskana auszufragen (er hat ihn am Dialekt als Toskaner erkannt); und dann das nicht weniger abrupte Auftauchen des Vaters von Dantes Jugendfreund Guido Calvacanti, der sich nach seinem - mittlerweile verstorbenen - Sohn erkundigen möchte. Ich zitiere die beiden Übergangsstellen. Zunächst das Ende des Gesprächs zwischen Vergil und dem Erzähler; dann das unvermutete Auftauchen Farinatas:

E io: „Buon duca, non tegno riposto a te mio cuor se non per dicer poco, e tu m'hai non pur mo a ciò disposto“. „O Tosco che per la città del foco vivo ten vai così parlando onesto, piacciati di restare in questo loco.
„Mein treuer Meister,“ sprach ich, „nicht verborgen Bleib dir mein Herz; es ist nur Schweigsamkeit, Die du mir anempfahlst erst diesen Morgen.“ „Tusker! Der in die Flammenstadt zu zweit In ehrendem Gespräch und lebend kam, Verweil, ich bitte dich, hier kurze Zeit. ${ }^{5}$

Dann folgt die Unterbrechung seines Gesprächs über die politischen Geschehnisse in Florenz nach seinem Tod; Calvacanti erhebt sich, langsam, in kontrastierender Bewegung, aus seinem Sarg:

„S’ei fur cacciati, ei tornar d'ogne parte“, rispuos' io lui, „l'una e l'altra fiata;
Drauf ich: „Wenn auch verjagt, sie kehrten frei Zweimal zurück, drum prüfe überlegend:

3 Vgl. hierzu Judith Kasper: Der traumatisierte Raum. Insistenz, Inschrift, Montage bei Freud, Levi, Kertész, Sebald und Dante, Berlin 2016, 198-201.

4 Auerbach, Mimesis (Anm. 1), $174 \mathrm{f}$.

5 Dante Alighieri: La Divina Commedia, hrsg. von Emilio Pasquini, Antonio Quaglio, Mailand 1988, Bd. 1, Inf. X, 19-24. Für die Übersetzung vgl. Dante Alighieri: La Commedia, übers. von Robert Zoozmann, Leipzig 1910. 
ma i vostri non appreser ben quell'arte“. Allor surse a la vista scoperchiata un'ombra, lungo questa, infino al mento: credo che s'era in ginocchie levata.
Ob diese Kunst den Deinen eigen sei?“ Hier hob sich neben ihm, sich langsam regend, Ein andrer Schatten aufrecht bis zum Kinne, Sich scheinbar auf den Knien hochbewegend. ${ }^{6}$

Bei der unerhörten Kunst des Trennens und Verbindens, die Auerbach hier rühmt, bleibt ein Moment - und zwar das auffälligste - unerwähnt: der Vers. Es ist ja vor allem der Vers, hier also die Wiederkehr eines Reims, der sich mit anderen Reimen zu dem äußerst dichten Geflecht der Terzine verbindet, der die Kontinuität dieser Übergänge nicht bloß ermöglicht, sondern garantiert. Es ist ja eine Eigentümlichkeit der terza rima, dass in ihr ein reimloser Übergang schlechterdings nicht möglich ist. Wie hart auch immer das aufeinander Folgende gefügt sein mag, wie verschieden in Stimmung, Gestus und Ausdruck: immer wird ein verbindender Klang aus dem Vorherigen mit hinübergenommen; phonische Protention und Retention verschränken sich unentwegt, so dass es nie zu dem jähen Abbruch kommen kann, von dem Auerbach ausgeht. ${ }^{7}$ Die Versordnung hält alles zusammen. Eigentlich kann es dort, wo gereimt wird, nicht zum Trauma kommen; oder anders: wo gereimt wird, hat die ,Durcharbeitung“ des Traumas je schon begonnen. ${ }^{8}$

Das führt auf ein grundsätzliches Problem. Auerbach zeigt sich in einer geradezu befremdlichen Weise indifferent gegenüber dem Unterschied von Vers und Prosa. Bereits das berühmte Einleitungskapitel, Die Narbe des Odysseus, ignoriert ihn, obwohl er sich aufdrängt. Denn was würde die Zuhörer wohl stärker in die zeitdimensional blinde, immerwährende Gegenwart der homerischen Erzählung verzaubern als der Umstand, dass sie gesungen, also in einer Art melodischem Sprechgesang vorgetragen wurde? Musik macht vergesslich; das gilt auch für Verse. Hochdeterminierte Gebilde wie ein Gedicht von Rilke oder die Schlusschöre von Faust II kann man sich zwar gut merken, aber es fällt schwer, zu erläutern, worum es eigentlich geht. Verse und Melodien bilden Zusammenhänge in der Zeit, können jedoch zeitlichen Differenzen nicht

6 Ebd., Inf. X, 49-54.

7 Es gilt sogar in abgeschwächter Form von dem Übergang zwischen Inferno und Purgatorio: das stelle, auf dem das erste Buch endet, wird in dem Reim nele - crudele zu Beginn des Fegefeuers fortgesetzt. Außerdem enden alle Bücher auf stelle: ein großer identischer Reim hält das kosmische Gefüge zusammen.

8 Kasper (Anm. 3), 198: Die Commedia sei „zu lesen als ein[ ] erste[r] große[r] Text der Weltliteratur, in dem eine traumatische Erfahrung nicht in einem antiken, tragischen Modell aufgehoben wird, sondern zu einem letztlich unaufgelösten Spiel und Widerspiel der Wiederholung führt, als immer wieder neu sich stellende Unentschiedenheit zwischen der schieren Insistenz und Wiederkehr eines Unbewältigten und der Chance seiner Durcharbeitung mit dichterischen Mitteln." ,Durcharbeitung“ versteht sie als eine „sprachliche Geste, die nicht primär auf Abbildung ausgerichtet ist, sondern in der Auseinandersetzung mit dem Phänomen der Wiederkehr des Unbewältigten in produktiver Weise mit der Differenz arbeitet, die in jedem Schreib- und Sprechakt am Werk ist“ (ebd., 199). Die fundamentalste dieser Differenzen ist die gebundene Sprache. 
artikulieren. Was klingt, ist Gegenwart, auch wenn von Vergangenem die Rede ist. Der Reim oder die rhythmische Wiederholung führt weniger zur Trennung von Gegenwart und Vergangenheit als zur intensivierenden Verstärkung dessen, was in diesem Moment gesagt wird. ${ }^{9}$ Und was würde umgekehrt die ungeheure, religiös-geschichtliche Spannung, die die Genesiserzählung des Isaakopfers freisetzt, stärker zum Ausdruck bringen als die einfache Satzfolge dieses prosaischen, ungebundenen und lückenhaften Textes?

Auerbach betrachtet alle Texte, die er interpretiert, als Prosa. Seine Literaturgeschichte ist eine Literaturgeschichte der Prosa - auch dort, wo sie von Versen handelt. Gegen die formalen Möglichkeiten, die der Vers birgt, und gegen die spezifischen Wirkungen, die von ihm ausgehen, ist Mimesis vollkommen unempfindlich.

Dass dergleichen einem Literaturwissenschaftler wie Auerbach einfach nur passiert sein sollte, kommt eigentlich nicht in Betracht - zumal er sich an anderen Stellen nicht scheut, vom Vers zu sprechen. In der Schrift Dante als Dichter der irdischen Welt heißt es:

so enthüllen die hundert Gesänge, in dem Glanz der Terzinenreime, in immer erneutem Verschlingen und Lösen, die traumhafte Leichtigkeit und Unnahbarkeit des Vollendeten, das mühelos zu schweben scheint wie ein Reigen unirdischer Gestalten. ${ }^{10}$

Die Unterdrückung des Verses in Mimesis kann kein Versehen sein; der Autor verfolgt eine zwar nicht begründete und explizierte, nichtsdestoweniger aber kalkulierte Strategie.

Worin dieses Kalkül bestanden haben könnte, lässt sich nur vermuten - aber es lassen sich einige der Voraussetzungen und Konsequenzen beleuchten, die mit dieser ,prosaisierenden' Vereinseitigung einhergehen: 1. Auerbach betrachtet Literatur vor allem als Schrift. 2. Die zentrale Organisationsform des schriftlichen Textes ist die Syntax. 3. Verse betonen die Gegenwart des Vollzugs innerhalb einer geschlossenen Form; Prosa dagegen einen offenen, häufig mehrperspektivischen Motivationszusammenhang. - Ich will versuchen, diese Bestimmungen im Einzelnen durchzugehen.

Zunächst: Man kann sicherlich nicht sagen, dass Prosa und Schrift einfach dasselbe seien; dennoch dürfte die Schrift so etwas wie ein natürliches Gravitationszentrum der Prosa darstellen. Verse reichen in die vorliterarische Frühgeschichte der Menschheit zurück und sind nicht zuletzt mnemotechnischen Ursprungs. Zwar liest man immer wieder, dass die Prosa recht eigentlich der mündlichen Rede entstamme; gerade diese aber bedarf der schriftlichen Fixierung, weil sie in sich nur wenig Gedächtnishalt findet. Das Mündliche bedarf der Schriftform, um literaturfähig zu werden. Alfred Döblins

9 Vgl. Wolfram Ette: Thesen zur Sprachähnlichkeit der Musik, in: Christian Grüny (Hrsg.): Musik und Sprache. Dimensionen eines schwierigen Verhältnisses, Weilerswist 2012, 43-50.

10 Erich Auerbach: Dante als Dichter der irdischen Welt, 2. Aufl., Berlin/New York 2001, 167. 
bekannte Formulierung, „dass man im Westen schreibt wie man spricht“, ${ }^{11}$ setzt gerade eine leicht zu erlernende und rasch zu vervielfältigende Schrift als Medium der Kodifizierung und Archivierung des quasi-Mündlichen voraus. Deswegen konnte sich die Prosa erst dann mit Macht entfalten - und das heißt auch: von der antiken Rhetorik als Lehre der mündlich vorzutragenden Prosa emanzipieren -, als der Buchdruck sich weiter und weiter verbreitete.

Was den Klang als wichtigstes Organisationsprinzip von Versen anlangt, so hat seine Prävalenz unterschiedliche Folgen. Einmal ist - Walter Ong hat das deutlich gemacht - in schriftlosen oder schriftarmen Kulturen das Verhältnis des gesprochenen Wortes zur Sache kein zeichenhaftes, sondern ein magisches.

For anyone who has a sense of what words are in a primary oral culture, or a culture not far removed from primary orality, it is not surprising that the Hebrew term dabar means, word' and ,event ' [...], that among ,primitive“ (oral) peoples generally language is a mode of action and not simply a countersign of thought [...]. Neither is it surprising that oral peoples commonly, and probably universally, consider words to have great power [...]. The fact that oral peoples commonly and in all likelihood universally consider words to have magical potency is clearly tied in, at least unconsciously, with their sense of the word as necessarily spoken, sounded, and hence power-driven. ${ }^{12}$

Die Sache wird nicht bezeichnet, sondern beschworen; sie ist in gewisser Weise gegenwärtig wie durch eine Zauberformel. Wenn Demodokos in der Odyssee vom Untergang Trojas singt, so ist im Klang der Worte, die die Sache verkörpern, das Ferne nah und das Vergangene gegenwärtig; deswegen verliert Odysseus beim Hören der Gesänge seine Fassung. ${ }^{13}$ Das ungeheure Ansehen der antiken Rhapsoden und Rhetoren, die Berichte über die außerordentlichen Wirkungen des gesprochenen Wortes deuten alle in diese Richtung.

Damit hängt zusammen, dass man Verse vor allem in fortlaufender Gegenwart wahrnimmt. Der zeitliche Horizont ist, wenn man Verse hört, eher klein. Die Assonanzen rhythmischer oder klanglicher Art, die den Vers gliedern, entfalten ihre Wirkung vor allem auf kurze Distanz; hier freilich lassen sie einen äußerst dichten Zusammenhalt entstehen. Aber bereits die terza rima von Dantes Commedia lässt sich kaum analytisch-trennscharf hören; eher hinterlässt sie einen allgemein-atmosphärischen Eindruck. Das hat zur Folge, dass syntaktische Strukturen im Vers in der Regel zu einer gewissen Einfachheit tendieren. Es muss nicht so sein wie in den meisten Psalmen,

11 Alfred Döblin: Die drei Sprünge des Wang-Lun. Chinesischer Roman, München 2007, 288.

12 Walter J. Ong: Orality and Literacy, New York 1982, 32.

13 Homer: Odyssee VIII 487-520, nach: Homer: Ilias \& Odyssee. Zweisprachige Ausgabe, übers. von Johann Heinrich Voss, Frankfurt a. M. 2008, 1009. Wenn es dann heißt: „Aber Odysseus / Schmolz in Wehmut, Tränen benetzten ihm Wimpern und Wangen. / Also weinet ein Weib und stürzt auf den lieben Gemahl hin, / Der vor seiner Stadt und vor seinem Volke dahinsank“ (ebd., 521-524), so wird ja suggeriert, dass ein bloß Vorgestelltes ihn ebenso betrifft wie ein wirkliches Ereignis. 
den Bluestexten oder bei Trakl, wo Vers- und Satzgrenze fast immer übereinstimmen; dennoch gibt der Vers dem Satz sein natürliches Maß vor. Wo, wie zum Beispiel bei Rilke oder Benn, Versform und Satzform in einem ästhetisch auffälligen Missverhältnis stehen, dürfte es vor allem eine Provokation, Dehnung und Belastung der hergebrachten Versform das Ziel sein.

Umgekehrt ist die Syntax der Prosa liebstes Kind. Auerbachs Fähigkeit, dieses Organisationsprinzip der Prosa zu erfassen und zu beschreiben, ist beeindruckend; und das gilt nicht nur für so komplexe Formen wie die Anrede Don Quijotes an die vermeintliche Dulcinea von Toboso sondern gerade auch dort, wo es auf den ersten Blick herzlich wenig zu beschreiben gibt: in den primitiven Satzfolgen eines Gregor von Tours, des heiligen Franziskus oder des altfranzösischen Rolandsliedes. Gerade aus dem, was uns rückständig, lückenhaft und in vielem Betracht langweilig vorkommt, holt er heraus, was an epochaler Wirklichkeitswahrnehmung hinter dem jeweiligen Text steht - und dies mit allen Unterschieden, die es im Einzelnen geben mag: Zwischen den kunstvollen, dramatisch eingesetzten Parataxen eines Augustinus, den monoton aufeinander folgenden Satzpanels des Alexiusliedes, den schwebend nebeneinander gesetzten Pointen Montaignes und den mit genialischer Betriebsamkeit ausgeworfenen Aufzählungen Balzacs liegen Welten; das, was über die Lücken zwischen den Sätzen oder Satzteilen adressiert wird, ist je und je etwas anderes.

\section{Prosa und Neuzeit}

Über dem Rausch der Differenzen, die Auerbach zu machen imstande ist, geht aber tendenziell eine große Entwicklungslinie verloren - vor allem der große Funktionswandel, den die erzählende Prosa auf dem Weg in die Moderne durchlaufen hat, in deren Verlauf sie den Vers in eine Nischenexistenz verbannte und zu der verbindlichen literarischen Ausdrucksform wurde. Hält man sich an die Kapitelfolge von Mimesis, so erfolgt der Durchbruch einer emanzipierten Prosa, die zu einer säkularen Wirklichkeitsdarstellung willens und in der Lage ist, im Decamerone. Boccaccio, so lesen wir in Mimesis, aber auch schon in Auerbachs früher Schrift über Die Technik der Frührenaissancenovelle in Italien und Frankreich, ist der erste selbstbewusste Prosaiker, der souverän über die Breite der mit sich verflochtenen Wirklichkeit verfügt. Alles wird hier „zu greifbarer Nähe, vielfältiger Lärm dringt hinein und die unbewegten Themen beginnen sich untereinander und mit der sinnlichen Welt zu verknüpfen; die Bilder wechseln, verknüpfen, kontrastieren“; es gelinge Boccaccio, „das Tatsachenmaterial in Bewegung zu bringen, daß es zu tanzen scheint. “14 In den analytischen

14 Erich Auerbach: Zur Technik der Frührenaissancenovelle in Italien und Frankreich, 2. Aufl., Heidelberg 1971, 44, 62. 
Partien des Boccaccio-Kapitels von Mimesis hebt Auerbach vor allem die Verfügung über das Tempo und den in eine einzige Bewegung zusammengefassten Perspektivreichtum „hypotaktischer Formen“ hervor, „wobei die von Boccaccio überhaupt sehr viel verwendeten Partizipialkonstruktionen die Hauptrolle spielen. “15 Bezogen auf die historische Konstruktion Auerbachs heißt das: Boccaccio erntet im Medium einer „Prosa der Welt“16, was Dante in einer letzten, selbstzerstörerischen Übersteigerung des figuralen, also theologischen Realismus des Mittelalters - und noch befangen im Vers - gesät hatte. „Ohne die Komödie hätte der Decamerone nie geschrieben werden können [...] die figurale Einheit der irdischen Welt ist in dem Augenblick zerbrochen, wo sie, bei Dante, volle Beherrschung der irdischen Wirklichkeit gewonnen hatte; die Beherrschung der Wirklichkeit blieb errungen“. ${ }^{17}$

Aber was heißt ,Prosa der Welt‘? Dass der Begriff der Prosa sowohl auf die Verfassung von Texten wie auf einen bestimmten, eben ,prosaischen' Weltzustand bezogen werden kann, ${ }^{18}$ deutet darauf, dass die Veränderung des Verhältnisses von Vers und Prosa selbst das Symptom eines veränderten Weltverhältnisses ist: eines prinzipiell anderen - und eben moderneren - Wirklichkeitsverständnisses, das sich in verschiedenen Phasen und Schüben seit dem Ende des Mittelalters Bahn brach. Wenn Auerbach sagt, dass die Literatur seit der Antike immer realistischer geworden sei, so hat er irgendwie recht; aber es handelt sich um einen ,Fortschritt‘, in dessen Verlauf sich der Begriff der Wirklichkeit selber qualitativ verändert - womöglich mehr als nur einmal. Die Phase zwischen Mittelalter und Neuzeit bezeichnet einen dieser Sprünge; und was die literarischen Formen anlangt, so drückt er sich unter anderem im unaufhaltsamen Siegeszug der Prosa aus.

Das veränderte Wirklichkeitsverständnis, das hier zutage kommt, lässt sich in einer Weise als anderes Verhältnis zur Zeit, insbesondere zur Zukunft darstellen. Sehr komprimiert ausgedrückt:

1. Die unbekannte Zukunft erregt nicht mehr Angst, sondern Neugierde - so jedenfalls hat es Blumenberg in seinen Studien über den Prozess der theoretischen Neugierde beschrieben, in dessen Verlauf die von Augustinus zur Todsünde

15 Auerbach, Mimesis (Anm. 1), 199.

16 Georg Wilhelm Friedrich Hegel: Vorlesungen über die Ästhetik I, in: ders.: Werke in 20 Bänden, hrsg. von Eva Moldenhauer, Karl Markus Michel, Frankfurt a. M. 1986, Bd. 13, hier: 199.

17 Auerbach, Mimesis (Anm. 1), 210, 218. Das Zitat fährt fort: , aber die Ordnung, in die sie [bei Dante] gefaßt war, ist nun verloren und es trat zunächst nichts an ihre Stelle.“ Das bezweifle ich, wie aus dem folgenden deutlich werden soll.

18 „Offenbar steckt im pejorativen Prädikat ,prosaisch“ eine Metonymie; sie überträgt die Gattungsbezeichnung ,Prosa' auf die profane, kunstferne Wirklichkeit, auf die sie stofflich festgelegt wird, und transformiert so den formalen in einen materialen Begriff“. Astrid Arndt, Christoph Deupmann: Poetik der Prosa. Zur Reflexionsgeschichte und Topik des Prosadiskurses, in: Astrid Arndt, Christoph Deupmann, Lars Korten (Hrsg.): Logik der Prosa. Zur Poetizität ungebundener Rede, Göttingen 2012, 19-34, hier 28. 
gemachte curiositas zur Rechtfertigung des ungezügelten weltlichen Forschungsdranges wurde. ${ }^{19}$

2. Peu à peu verlieren die Zielursachen, die in Antike und Mittelalter für die Steuerung von Prozessen verantwortlich waren, ihre Bedeutung und die causa efficiens, also die mechanische Kausalität (die nicht teleologisch auf die Zukunft ausgreift), wird zur privilegierten Form des Zusammenhangs zwischen den empirisch Seienden; entsprechend ist der Mensch nicht mehr Teil eines vorgeordneten Heilsplans, sondern kann, indem er auf die Ursachen Einfluss nimmt (zum Beispiel durch experimentelle Forschung), die Wirkungen verändern und sich als jemand entwerfen, der die Zukunft gestaltet anstatt ihr ausgeliefert zu sein. ${ }^{20}$

3. Das individuelle Leben erscheint auf den kreatürlichen Rahmen von Geburt und Tod zurückgeworfen. Bei Auerbach tritt dieses Moment eigenartigerweise erst in dem Kapitel über Madame du Chastel hervor; zur systematischen Entfaltung gelangt es dann bei Montaigne. Der Begriff der condition humaine, der dem Montaigne-Kapitel von Mimesis seinen Titel gegeben hat, bedeutet ja nichts anderes als den Versuch, die Eigenart des menschlichen Lebens ohne transzendent gegründete Wesensdefinitionen zu bestimmen; er bezeichnet eine rein säkulare Wesensbestimmung des Menschen - so wie sich in den Essais nur unbedeutende Spuren der katholischen Doktrin finden lassen: „der Geist der ,Essais“ ist vollkommen unchristlich, denn sie handeln vom Tode, als gäbe es keine Erlösung und keine Unsterblichkeit.“21 Das aber ist bereits im Decamerone der Fall. Die Rahmenerzählung zeigt deutlich die Ausgangssituation dieses Erzählens: Das christlich-mittelalterliche Ordnungssystem ist zusammengebrochen; die Pest

19 Hans Blumenberg: Die Legitimität der Neuzeit, Frankfurt a.M. 1988, 261-528. Augustinus Verwerfung der Neugierde als „triebhafte Selbstgenügsamkeit des Sichüberlassens an die Welt der Erscheinungen“ (ebd., 361) findet sich in den Confessiones, V 3, X 35; die erste philosophisch prägnante „Anerkennung des menschlichen Wissensdranges in seiner unbeschränkbaren Dynamik“ (ebd., 416) lässt sich, Blumenberg zufolge bei Nikolaus von Kues festhalten.

20 Freisetzung der causa efficiens: Martin Heidegger: Die Frage nach der Technik, in: ders.: Vorträge und Aufsätze, Pfullingen 1954, 13-44. Das berühmte Billardkugel-Beispiel, das die Verselbständigung der Wirkursache auf ein traditionsmächtiges Bild gebracht hat, findet sich bei David Hume. Dort heißt es am Ende: „Die Bewegung der zweiten Billiardkugel ist ein völlig verschiedenes Ereignis von der Bewegung der ersten; auch ist in der einen nichts enthalten, das die leiseste Andeutung der anderen lieferte“ (David Hume: Eine Untersuchung über den menschlichen Verstand, übers. von Raoul Richter, hrsg. von Jens Kulenkampff, Hamburg 1993, 39). Das Programm neuzeitlicher Wissenschaft, also Einflussnahme auf die Ursachen, damit die Befreiung von Zielursachen und letzten Prinzipien ist das Programm von Bacons Novum Organon. Vgl. hierzu Klaus Heinrich: tertium datur. Eine religionsphilosophische Einführung in die Logik, Basel u. a. 1987. Zu diesem Komplex insgesamt: Jan Friedrich: Zusammenspiel mit der Natur. Wirklichkeit und Utopie einer spielerischen Technik, Weilerswist 2015, 69-100.

21 Erich Auerbach: Der Schriftsteller Montaigne, in: ders.: Die Narbe des Odysseus. Horizonte der Weltliteratur, hrsg. und eingeleitet von Matthias Bormuth, Berlin 2017, 37. Zum Begriff der Kreatürlichkeit: Auerbach, Mimesis (Anm. 1), 237-239. 
in Florenz, nach deren Ende Boccaccio seinen Novellenzyklus begann, hat alle überlieferten Werte mit sich gerissen; die Balance von Diesseits und Jenseits ist auseinandergerissen und hat einer rücksichtslosen Diesseitigkeit Platz gemacht. Das ist der dunkle Grund, auf dem das alles andere als problemfreie Gebäude des Decamerone errichtet wird..$^{22}$ Das Decamerone stellt den Versuch dar, dem kreatürlich auf sich zurückgeworfenen, zwischen den Polen von Eros und Todesdrohung gespannten Menschen ein zivilisatorisches Ordnungsangebot zu machen, das auf der Fähigkeit gebildeter Menschen, zu erzählen, beruht; das Erzählen ist die einzige Möglichkeit, in einer Welt, die kein metaphysisches Obdach mehr bietet, Orientierung zu gewinnen.

4. Und psychologisch tritt der Eros, um den die Literatur vom dolce stil nuovo bis Boccaccio kreist, als entscheidende Triebkraft eines nicht vom Ende her, sondern durch Anfang und Mitte bestimmten Geschehens hervor, die es erlaubt, den Zufall ins Geschehen zu integrieren.

Auf diesen letzten Punkt muss ich ein wenig ausführlicher eingehen. Der Eros ist seit der Antike eine Schicksalsmacht. Aber er unterscheidet sich in einigen wesentlichen Punkten von den klassischen Providenzvorstellungen. Einmal wird er nicht von oben verhängt; sein Ursprung geht auf keine transzendente Machtinstanz zurück; seine unwiderstehliche Verfügung über Menschen und Götter, wie es bei Hesiod heißt, hat vielmehr den Grund, dass er aus dem Inneren des Lebendigen hervorbricht. ${ }^{23}$ Auch wenn er dem Menschen nicht in der Weise ,gehört', dass er nach Belieben über ihn verfügen könnte, so gehört er doch in andere Weise $z u$ ihm als ein fern von der Immanenz seines individuellen Lebens gefällter Götterentscheid. Das Schicksal des Ödipus wird vor seiner Geburt beschlossen, und ob es Odysseus gestattet wird, nach Hause zurückzukehren, hängt weniger von ihm ab als von den Sympathien einzelner Götter und den Machtverhältnissen auf dem Olymp ab. Das Schicksal des Begehrens dagegen

22 Vgl. ebd., S. 220 f. In dieser kritischen Würdigung Boccaccios fallen Begriffe wie ,flüchtig‘, ,abenteuerlich', ,sentimental‘, ,rührend‘, ,unklar‘ und ,unsicher‘. Das lässt sich dahin zusammenfassen, dass Boccaccio zum wirklichen Ernst nicht imstande sei. Die Allgegenwart eines verheißungslosen Todes und das durchaus ,tragische“ Getriebensein von der erotischen Leidenschaft, die das Erzählen zur überlebensnotwendigen „Erfrischung“ (rifrigerio) macht, bleiben dabei unberücksichtigt. Vgl. Giovanni Boccaccio: Vorrede, in: ders.: Das Dekameron, München 1965, 7.

23 Hesiod: Theogonie, hrsg., übers. und erläutert von Karl Albert, Sankt Augustin 1985, 120-122. Deswegen sind ihm auch die Götter unterworfen - ein Zug, der vor allem bei Euripides hervortritt. „Nicht nur die irdischen Männer kommt die Liebe an / Und ird'sche Frauen; nein, der Götter Seelen selbst / Erregt sie und sie wandert übers Meer sogar. / Auch Zeus der Allgewaltige vermag es nicht, / sie abzuhalten, weicht und giebt ihr willig nach.“ Euripidis Perditarum Tragoediarum Fragmenta, hrsg. von August Nauck, 2. Aufl, Leipzig 1885, Bd. 3, fr. 431. Zitiert nach Werner Nestle: Euripides. Der Dichter der griechischen Aufklärung, Stuttgart 1901, 261. Zur Entdeckung des Eros als tragischer Macht vgl. Jaqueline de Romilly: La Modernité d'Euripide, Paris 1986, 43 f. 
ereilt die individuelle Existenz aus der Mitte ihres Lebens heraus - ,von unten', wenn man das so sagen darf.

Es tritt hinzu, dass am Anfang eines solchen Schicksals ein Zufall steht - der Zufall einer Begegnung, an der sich das Begehren entzündet. Seit den Anfängen des erotischen Erzählens ist immer wieder versucht worden, diese Kontingenz des Anfangs zu verschleiern; durch die Erzählweise medias in res bei Heliodor etwa; oder durch den Zahlenzauber, den Dante und Petrarca bemühen, um die Begegnung mit Beatrice/ Laura vom Anschein, dass es auch hätte anders sein können, zu lösen und als quasi heilsgeschichtliches Ereignis zu etablieren. Wo aber der Anfang zufällig ist, könnte es auch das Ende sein; potenziell beschreibt der Eros eine Schicksalsmacht ohne Telos; eine fast richtungslose Übermacht, die das Ende nicht in sich enthält, ohne dadurch aufzuhören, eine Übermacht darzustellen. Daher rührt die massive Rationalisierung des erotischen Geschehens durchs Schema der Prüfung - im antiken Liebesroman, aber auch in den höfischen Versepen -, und die Erhebung der Tyche (später dann der Fortuna) zu einer dirigierenden Schicksalsmacht im Hellenismus, deren Prinzip gerade die Blockade einer analytischen Trennung von Zufall und Vorsehung ist: ihre Unauflöslichkeit, die im Zweifel immer zugunsten der Vorsehung ausfällt, ist hier zum mytho-theologischen Programm geworden.

Aus dieser seltsam schiefen Lage wird das Erzählen erst durch Boccaccio befreit. Die relativ starke, wenn auch nicht ausschließliche Konzentration der Novellen des Decamerone auf erotische Sujets legt nicht allein eine Liebes- und Naturordnung frei, die der religiösen Ordnung des Mittelalters entgegengesetzt wird, ${ }^{24}$ sondern auch ein bislang verborgenes Fundament säkularen Erzählens. Boccaccio verfolgt die Windungen und Wendungen eines im Begehren begründeten, im Prinzip nicht voraussehbaren, nicht durch Vorsehung gesteuerten Geschehens; auf einem letalen Nullpunkt der Gesellschaft vertraut er sich und die Leser`innen seines Buchs Prozessen an, von denen je und je nicht gesagt werden kann, wohin sie führen; die Erzählwege, die er in seine erotische Karte des Mittelmeerraumes einträgt, weisen prinzipiell ins Offene.

Das diesen vier Aspekten, in denen die beginnende Neuzeit sich selbst entwarf, Gemeinsame ist ,Geschichtlichkeit': die Öffnung des Zukunftshorizonts, sei es in der Form, dass er als Sphäre des von Menschen zu Gestaltenden vor Augen trat; oder so, dass er als Entfaltungsraum eines nicht teleologisch konzipierten Schicksals sich darbot. Für beides bot sich die Prosa als Entfaltungsmedium an; ihre Freisetzung ist der ästhetische Ausdruck der Emanzipation vom teleologischen oder sonst vom Ende her bestimmten Prozessschema.

24 Vgl. Auerbach, Mimesis (Anm. 1), 216 f. Die Einsicht, dass es vergebens ist, sich gegen die fundierende Triebmacht der Natur aufzulehnen, findet sich in den Einleitungen zum ersten und vierten Tag; dass die Liebe, wenn man sich ihr anvertraut, eine kulturbildende Kraft darstellt, zeigt sehr schön die von Auerbach (ebd.) angeführte Geschichte von Cimone (in: Boccaccio, Das Dekameron [Anm. 22], $392-403[5,1])$. 
Das ,gebundene، Prozessschema steht wiederum in einer eigentümlichen Konjunktion mit dem Vers. Was kann ,gebundene Rede“ anderes heißen, als dass der syntaktisch-semantische Sprachverlauf einem äußeren Schema unterworfen wird, das durch Wiederholung bestimmt ist? Im Schema der Wiederholung erscheint aber der Anfang als das vorweggenommene Ende, bzw. als ,Noch-nicht‘ (steresis) eines antizipierten Endes, das Ende aber als der Moment, in dem der Anfang zu sich selber zurückkehrt und zum Ursprung wird. Jeder Vers, der durch metrische Regelmäßigkeit gekennzeichnet ist, macht ein Versprechen für die Zukunft; jeder scheint ein Versprechen, das aus der Vergangenheit kommt, zu erfüllen; in der Wiederholung kreisen arché und télos umeinander wie in den Prozessen der entelecheia, die Aristoteles in der Physik analysiert. Der Vers macht Teleologie zur sinnlichen Erfahrung; er stellt eine Dauerberieselung durch eine teleologische Mikrostruktur dar, die die Hörer^innen in Bann schlägt, der Ursprung und Ziel unablässig ineinander konvertiert und die Wahrnehmung offener Prozesse maximal erschwert. ${ }^{25}$ Der Vers spricht - aber dafür in Form eines suggestiven Gewebes - unablässig Zukunftsgarantien aus; einer Epoche, die darauf angewiesen war, vermochte er Weltvertrauen, eine Form der Beherrschung der Zeitläufte durchs letztlich anthropogene Schema der Teleologie zu vermitteln. ${ }^{26} \mathrm{Im}$ Vers ist man geborgen; er ist ein Schutzraum, der vor dem Unbekannten und tendenziell Gefährlichen schützt. Prosa hingegen konnte sich durchsetzen, als diese Angst von den Menschen wich, oder durch andere Temporalsynthesen balanciert wurde. ${ }^{27}$

25 „Ursprung ist das Ziel“ (Karl Kraus, Schriften, hrsg. von Christian Wagenknecht, Frankfurt am Main 1989, Bd. 9, 68): Das ethische Programm, dem Karl Kraus sich verpflichtet sah, gilt auch für die gebundene Sprache, ja findet in ihr ein fast reflexionsloses Entfaltungsmedium. Seine berühmten Verse über den Reim „Er ist das Ufer, wo sie landen, / sind zwei Gedanken einverstanden“ (Karl Kraus, Der Reim, in: ders.: Ausgewählte Gedichte, München 1920, 41) realisieren das Schema: Ursprung - die Harmonie der Gedanken - und Ziel - das Ufer des Zusammenklangs - tauschen ihre Rollen, verschweben eigentümlich ineinander und bilden ein ästhetisches Korrelat der teleologischen Denkform.

$26 \mathrm{Zu}$ dem von Aristoteles zuerst konzeptualisierten Wechselbezug von physis und techne vgl. Hans Blumenberg: „Nachahmung der Natur“. Zur Vorgeschichte der Idee des schöpferischen Menschen, in: ders.: Ästhetische und metaphorologische Schriften, Auswahl und Nachwort von Anselm Haverkamp, Frankfurt a. M. 2001, 37: „Deshalb musste Aristoteles die Genesis z. B. des Hauses so erklären, daß der Architekt hier etwas zustande bringt, was die Natur ebenso entstehen lassen würde, das heißt: er muss sich dieses künstliches Gebilde als Naturprodukt vorstellen, um dann diese hypothetische Vorstellung nachzuahmen." Anhand der Physikvorlesung habe ich dies rekonstruiert, vgl. Wolfram Ette: Die Aufhebung der Zeit in das Schicksal. Zur „Poetik“ des Aristoteles, Berlin 2003, 17-21.

27 Martin von Koppenfels hat die These vertreten, dass das Mimesis zugrundeliegende, begrifflich nicht artikulierte, sondern motivisch umspielte Realismuskonzept etwas mit Trennung zu tun habe. Erst durch einen Verlust (das Exil, den Verlust der Mutter im Ödipuskonflikt) kann man dahin gelangen, die Wirklichkeit anzuerkennen - als etwas eben auch literarisch zu bewältigendes. Vgl. Martin von Koppenfels: Auerbachs Ernst, in: Poetica 45 (2013), 183-203. Ist es eine verwegene Analogie, zu vermuten, dass der Weg, der aus dem Schutzraum des vorneuzeitlichen Ursprungsdenkens - und seinem ästhetischen Korrelat des Verses - herausführte, eine systematisch vergleichbare Verluster- 


\section{Motivation von hinten - fortlaufende Motivation}

Nun hat es Prosa auch vor dem hier angedeuteten Epochenbruch gegeben; ein nicht geringer Teil von Auerbachs Analysen ist ja eben diesen Erscheinungsweisen vorneuzeitlicher Prosa gewidmet. Aber wenn es mit den Vermutungen, die ich hier äußere, seine Richtigkeit hat, dann hätte die Prosa durch den Epochenbruch ein Potenzial gewonnen, das sie zuvor nicht hatte. Dieses Potenzial möchte ich in Anlehnung an Auerbachs Zeitgenossen Clemens Lugowski die ,Kunst der fortlaufenden Motivation“ nennen.

In seiner Dissertation über Die Formen der Individualität im Roman analysiert Lugowski die frühneuzeitlichen - also im 16. Jahrhundert verfassten - Prosaromane Jörg von Wickrams. An dieser Prosa hält er verschiedene archaische Merkmale fest: eine lineare Anschauung, die gleichzeitige Handlungen nicht zulässt; eine Tendenz zu unverbundenen Aufzählungen; die Reduzierung von Personen auf ihre Handlungsfunktion und die Neigung zur identischen Wiederholung von Formulierungen und Handlungssegmenten. Das wichtigste Charakteristikum indessen, in dem sich alle anderen Eigentümlichkeiten dieser Prosa zusammenfassen, ist die Motivation von hinten. Damit ist gemeint, dass es zu einer Motivierung im psychologischen Sinn nicht kommt. Das psychologische Motiv ist das innerseelische Korrelat der freigesetzten und unabhängig gewordenen causa efficiens. Sein Nichtvorhandensein führt dazu, dass das Ergebnis in gewisser Weise immer schon da ist; niemals führt ein Prozess in kontinuierlicher und für uns nachvollziehbarer Weise zum Ergebnis. Lugowski schreibt:

Die Motivierung [...] liegt nicht in irgendeiner Prämisse, sondern in nichts anderem als der großartigen einfachen Selbstgenügsamkeit des ergebnishaft sich manifestierenden reinen Seins, das alles endlich-zeitlich Bewegte auf sich hin, in sich hinein zieht. ${ }^{28}$

Deswegen schreitet die Handlung in diesen Texten so stotternd und ruckhaft voran; deswegen kann mit sich wiederholenden Versatzstücken gearbeitet werden; deswegen können die Personen auf ihre Funktion für das Ergebnis reduziert werden -: weil es als unveränderliches Sein immer schon da ist und gar nicht entwickelt oder begründet werden muss; weil diese Prosa noch teilhat am Ursprungsdenken der Vormoderne das vom Vers eigentlich besser und suggestiver ausgedrückt werden kann.

fahrung dargestellt hat? Die geschichtsphilosophische Trauer, die in der klassischen Ästhetik den Begriff der Prosa umgibt, spricht dafür.

28 Clemens Lugowski: Die Form der Individualität im Roman [1932], Frankfurt a. M. 1976, 75. Lugowskis Gegenbegriff zu „Motivation von hinten“ ist der der „,vorbereitenden Motivation“. Ich habe das in ,fortschreitende“ Motivation geändert, weil mir der Charakter eines Motivationssystems, eines Zusammenhangs in Bezug auf einander ändernde Motivationsimpulse besser ausgedrückt zu sein scheint. 
Dieses Ursprungsdenken der Vormoderne wird von Lugowski auch Boccaccio zugeschlagen - meines Erachtens zu Unrecht. Es sind vor allem zwei Argumente, die Lugowski für die These bemüht, dass der Decamerone noch ganz der Welt des „mythischen Analogons“ zugehörig sei, das wesentlich durch die ,Motivation von hinten“ charakterisiert ist. Erstens erscheint die Liebe in den Novellen als Schicksalsmacht, der gegenüber die von ihr Erfassten nichts vermögen: Die Liebe

ist etwas, dem die Menschen verfallen, von dem sie überwältigt und beherrscht werden [...]. Diese Liebenden sind alle mehr Ergriffene als Ergreifende; ergriffen von einem verzehrenden Wahnsinn, und die Liebesbewegung geschieht in ihren reinsten Ausprägungen so unbedingt, unerbittlich und hemmungslos abrollend, daß es ihren Ablauf durch einen Menschen hindurch - das will hier nur sagen: durch etwas, das nicht Liebe ist - gar nicht zu geben scheint [...]. Sie [die Liebenden] bewegen sich nicht selbst, sondern werden bewegt, sie töten sich nicht, sondern ,es tötet sie [...] Lieben ist keine aktive Gerichtetheit [...], sondern nur in passivischen Wendungen zu umschreibendes Sein. ${ }^{29}$

Das ist richtig (wenngleich mir der tragische Akzent, den Lugowski ihm verleiht, falsch gesetzt zu sein scheint); und doch handelt es sich eben nicht um ein providentielles Schicksal, sondern um eines, das dem Zufall und der Kontingenz einen anderen Handlungsverlaufs Spielraum gewährt. Wie ließe sich etwa die siebte Geschichte des zweiten Tages noch irgend mit dem Schema des providentiellen Schicksals übereinbringen? Eine Frau gerät auf dem Weg zu ihrem zukünftigen Ehemann „durch eine Reihe von Ereignissen in einem Zeitraum von vier Jahren und an verschiedenen Orten neun Männern in die Hände“. ${ }^{30}$ Dieser Weg durch ca. 10000 Geschlechtsakte, die sie mit immer größerer Lust vollzieht, führt sie am Ende zwar ihrem Anvertrauten zu, aber in Form einer pragmatischen, bürgerlichen Lösung, die ihr Sicherheit gewährt. Der Ehemann erfährt nichts von alledem; er hält seine Frau für unberührt; ihr Weg zu ihm scheint ihm der einer mit allen Mitteln verteidigten Jungfräulichkeit, die das telos, ihn wiederzufinden, nie aus den Augen verloren hatte. Für ihn ist es ein Plot, der dem antiken Liebesroman entnommen sein könnte, für sie eine Kette von Begebnissen, deren jedes mit erotischer Präsenz und der Verheißung, dank ihrer das Ziel endgültig aus den Augen zu verlieren, verbunden ist. Zufälle reichen nicht aus, erst wenn man sich mit ihnen durch das Begehren identifiziert und die Zukunft durch erfüllte Gegenwart zum Verschwinden bringt, entstehen neue narrative Verläufe, die vom teleologischen Schema allenfalls notdürftig abgedeckt werden können.

Zweitens, so Lugowski, seien sowohl die Verabredungen der Erzähler`innen hinsichtlich der Novellensujets eines einzelnen Tages als auch die jeder Novelle vorangestellten Inhaltsangaben „spannungsvernichtend“31 - wenn anders ,Spannung“

29 Ebd., $33 \mathrm{f}$.

30 Boccaccio, Das Dekameron (Anm. 22), 139.

31 Lugowski (Anm. 28), 40. 
bedeute, dass das ,mythische Analogon' sich auflöse und von individuellen Figuren erzählt werde, die sich in nicht eindeutig voraussagbaren Abläufen bewegen. Die Dinge scheinen sich aber komplizierter zu verhalten. Durch den Umstand, dass die Sujetvorgaben am ersten und neunten Tag (plus in der jeden Tag abschließenden Erzählung des Dioneo) unbestimmt bleiben, wird ein Potenzial angezeigt, das für alle gilt; es ist die fortschrittlichere Form, der gegenüber die fixierende Festlegung als Konvention erscheint. Und was die Inhaltsangaben betrifft, so ist zum einen ihr Textstatus unklar: Werden sie von den Erzähler^innen ausgesprochen und gehört oder sind es herausgeberische Hinzufügungen? Zum anderen ist ihr Verhältnis zu den ihnen folgenden Erzählungen nicht so eindeutig, wie es bei Lugowski erscheint. Sie können lakonisch bis zur Unverständlichkeit sein, ${ }^{32}$ umgekehrt so ausführlich, dass man ebenfalls kein klares Bild des Erzählten bekommt, ${ }^{33}$ oder sie geben über den Ausgang der Geschichte gar keine Auskunft. ${ }^{34}$ Es sind in vielen Fällen weniger Inhaltsangaben als Teaser, die so wenig vorwegnehmen, dass sie eher spannungssteigernd als -vernichtend wirken. Sie entwerfen einen Erwartungsraum, in dem die Spannung sich entfalten kann.

Es ist nicht leicht zu sagen, warum Lugowskis Fähigkeit zur Textbeobachtung sich an Wickram so glänzend bewährt und an Boccaccio so auffallend scheitert. Ich vermute, dass der Dämon der Chronologie zu diesem Missverständnis geführt hat. Boccaccios Werk geht dem Jörg von Wickrams um 200 Jahre voraus. Also muss es irgendwie auch archaischer sein. Das verkennt die Ungleichzeitigkeit der Entwicklungen in Italien und Deutschland, sowie, dass die Genese der literarischen Neuzeit sich nirgendwo linear und störungsfrei vollzogen hat. In Italien erfolgte schon Ende des 14. Jahrhunderts ein rollback ${ }^{35}$ und was Deutschland betrifft, so wird die Vorgeschichte des höfischen Romans (inklusive ihrer prosaischen Fortwucherungen) von Lugowski ausgeblendet.

Es wäre nun eine eigene Aufgabe, zu zeigen, wie die ,Motivation von hinten“ so etwas wie einen Brennpunkt darstellt, auf den hin sich viele der vormodernen Texte, die Auerbach in Mimesis analysiert, bündeln lassen. Ich nenne aber zumindest einige Stichworte, die erkennen lassen, wie weit Lugowkis Konzept einer ,Motivation von hinten' als Textparadigma der Vormoderne reichen könnte: die Parzellierung des Geschehens in starke Gesten, die Auerbach am Rolandslied analysiert (gerade weil der figuraltheologische Ansatz in den Chansons de geste relativ schwach ausgeprägt ist); die chronikalische Lakonik des Bischofs Gregor aus der Merowingerzeit, der von den Ereignissen in seiner Gemeinde erzählt, ohne sie im geringsten zu verbinden; die Monotonie der Heiligenlegenden und Heiligenviten, die gleichfalls die berichteten Vorgänge faktografisch und verbindlungslos aneinanderreihen; aber auch, über das

32 Boccaccio, Das Dekameron (Anm. 22) 1,6; 1,7; 1,10; 3,5.

33 Ebd., 2,6; 5,10.

34 Ebd., 9,9.

35 Vgl. Auerbach, Frührenaissancenovelle (Anm. 14), 24-30. 
von Auerbach ausgewählte Corpus hinaus, die entwicklungslosen Handlungs- und Stilschemata der Märchen, in denen je schon alles feststeht. Mit Lugowski (und gegen Auerbach) wäre zu sagen: All dies ist kein Mangel, keine Defizienz einer noch unausgereiften Fähigkeit der Literatur zur Darstellung von Wirklichkeit, sondern Ausdruck eines eigenen, eben vorneuzeitlichen Wirklichkeitsbegriffs.

Wenn Auerbach den Unterschied zwischen Vers und Prosa verschleift, dann verschleift er damit den Unterschied zwischen zwei verschiedenen Wirklichkeitsbegriffen und den ihnen entsprechenden Formen des literarischen Realismus. Auf der einen Seite haben wir den letztlich religiös fundierten Realismus der älteren Literatur, der sich teleologisch, figural oder durch ,Motivation vom Ende‘ her zur Geltung bringt und der sich intentional vor allem im Vers erfüllt; auf der anderen Seite findet sich der eigentlich säkulare Realismus, der sich schrittweise ab etwa 1300 entfaltet und strikt an die Prosa gebunden ist. Der ältere Realismus kann höchst ausdrucksstark im einzelnen, gestischen Detail sein, aber er gelangt nicht zu einer motivischen Verflechtung. Es ist nicht nötig, einen Zusammenhang herzustellen, weil alles so ist, wie es sein soll, und weil alles vom Ende her entschieden ist oder sich in der theologischen ,Figur‘ auflöst. Verse vermögen über das Diskontinuierliche dieser Darstellungsweise hinweggeleiten; ihr ,voller Ton', die Suggestion durchlaufender Präsenz und die dichte Textur klanglich hergestellter Intentionalitätsbeziehungen füllen wie von selbst die Lücken der Texte.

Die säkulare Prosa dagegen hat das von sich aus zu leisten. Daher rührt die sprunghafte Entwicklung syntaktischer Souveränität in den Volkssprachen während der Renaissance. Der Rückgriff auf die Tradition der antiken Rhetorik stellt dabei nur das Mittel zum modernen Zweck dar: Verfügungsmacht über den säkularen Raum zu erlangen, in dem sich eine Fülle von Motiven, Perspektiven und Zuständen in hochindividuierter Form überkreuzen, einander widersprechen und miteinander interferieren. Lugowski spricht in diesem Zusammenhang sehr schön vom „Zickzack“ des Weltlaufs ${ }^{36}$. Dem misst sich die neue Prosa an. Der Satz und die Sätze werden zum Schauplatz einer solchen Szenerie, in der Verschiedenstes durcheinanderwirkt und auf ein unbekanntes oder nur in Umrissen erkennbares Ziel zusteuert. Aus den Momentaufnahmen der älteren Prosa, die vom Ergebnis nur durch den zeitlichen Abstand getrennt sind, werden fluktuierende, rekursiv organisierte, multiperspektivische und flexibel prozessierende Systeme, die im Verlauf ihre eigene Zukunft entwerfen und ihre eigene Vergangenheit modifizieren. Geschwindigkeit, Zeiten, Zustände und Ziele werden in der neuen Prosa in einer Komplexität miteinander verschaltet, zu der der Vers nicht in der Lage ist und nicht in der Lage zu sein brauchte, weil die Vorstellung von Wirklichkeit es nicht erforderlich machte.

36 Lugowski (Anm. 28), 73. 
